\title{
A hybrid configuration interaction treatment based on seniority number and excitation schemes
}

\author{
Diego R. Alcoba, ${ }^{1}$ Alicia Torre,${ }^{2}$ Luis Lain, ${ }^{2, a)}$ Ofelia B. Oña, ${ }^{3}$ Pablo Capuzzi, ${ }^{1}$ \\ Mario Van Raemdonck, ${ }^{4}$ Patrick Bultinck, ${ }^{4}$ and Dimitri Van Neck ${ }^{5}$ \\ ${ }^{1}$ Departamento de Física, Facultad de Ciencias Exactas y Naturales, Universidad de Buenos Aires and \\ Instituto de Física de Buenos Aires, Consejo Nacional de Investigaciones Científicas y Técnicas, Ciudad \\ Universitaria, 1428 Buenos Aires, Argentina \\ ${ }^{2}$ Departamento de Química Física, Facultad de Ciencia y Tecnología, Universidad del País Vasco, Apdo. 644 \\ E-48080 Bilbao, Spain \\ ${ }^{3}$ Instituto de Investigaciones Fisicoquímicas Teóricas y Aplicadas, Universidad Nacional de La Plata, CCT La \\ Plata, Consejo Nacional de Investigaciones Científicas y Técnicas, Diag. 113 y 64 (S/N), Sucursal 4, CC 16, \\ 1900 La Plata, Argentina \\ ${ }^{4}$ Department of Inorganic and Physical Chemistry, Ghent University, Krijgslaan 281 (S3), 9000 Gent, Belgium \\ ${ }^{5}$ Center for Molecular Modelling, Ghent University, Technologiepark 903, 9052 Zwijnaarde, Belgium
}

(Received 3 September 2014; accepted 9 December 2014; published online 31 December 2014)

\begin{abstract}
We present a configuration interaction method in which the Hamiltonian of an $N$-electron system is projected on Slater determinants selected according to the seniority-number criterion along with the traditional excitation-based procedure. This proposed method is especially useful to describe systems which exhibit dynamic (weak) correlation at determined geometric arrangements (where the excitation-based procedure is more suitable) but show static (strong) correlation at other arrangements (where the seniority-number technique is preferred). The hybrid method amends the shortcomings of both individual determinant selection procedures, yielding correct shapes of potential energy curves with results closer to those provided by the full configuration interaction method. (C) 2014 AIP Publishing LLC. [http://dx.doi.org/10.1063/1.4904755]
\end{abstract}

\section{INTRODUCTION}

It is well known that the full configuration interaction (FCI) method provides the exact solutions of the Schrödinger equation for an $N$-electron system in a given Hilbert space. Unfortunately, its factorial growth with respect to the number of electrons and the number of basis set orbitals demands a prohibitive computational cost. One of the most popular approaches used to reduce this computational cost is to limit the number of Slater determinants in the configuration space; this idea constitutes the foundation of the configuration interaction (CI) methods. This technique requires to select, according to a determined criterion, the Slater determinants in which the $\mathrm{N}$ electron Hamiltonian is projected. Most often, this selection is performed in terms of the number of spin-orbitals excited with respect to a given reference determinant. However, more recently an alternative CI procedure has been proposed; in this case, the selection of the Slater determinants is accomplished according to the seniority number of the determinants used to project the Hamiltonian ${ }^{1-5}$ (the seniority number has been defined as the number of singly occupied orbitals in a determinant). ${ }^{6,7}$ On the other hand, the electronic correlation energy is usually decomposed into two components which have been denominated as dynamic (weak) and static (strong) correlations. $^{8-12}$ The correlation is essentially dynamic when a single-determinant reference is a good zeroth-order wave function. The static (nondynamic) correlation is important in

\footnotetext{
a)Author to whom correspondence should be addressed. Electronic mail: qfplapel@lg.ehu.es
}

systems with strong multireference character and necessarily requires the use of wave functions composed of multiple Slater determinants, even for suitable zeroth-order descriptions. Systems with near-degenerate ground states, molecules with stretched bonds, radicals, complexes of transition metals, and so forth are examples which fit this correlation model, which have also been found in bond-breaking processes and in the description of superconductivity and other properties of materials.

The performance of both excitation- and senioritynumber-based selection criteria in CI expansions to describe $N$-electron systems has been tackled in Ref. 1 which reports studies of atoms and molecular species in which either dynamic or static correlation is dominant. The conclusions reported in that reference indicate that the excitation-based configuration selection procedure is more efficient than the seniority-based approach when the system presents essentially dynamic correlation. However, in those situations where the static correlation is important, the seniority-number-based selection procedure leads to better results. In the description of dissociation processes or bond breaking, one often finds molecular systems that show mainly dynamic correlation at arrangements near the equilibrium distances but present static correlation at stretched arrangements. A global study of such systems raises the problem of choosing one of the two above mentioned CI expansion construction criteria. The aim of this work is to propose a method that combines both CI expansion types, providing a framework that accounts efficiently for both types of correlation. In our procedure, the $N$-electron Hamiltonian is projected on a set of Slater determinants which 
possess a determined seniority number plus the additional Slater determinants needed to complete a given excitation level with respect to a reference. The method turns out to be suitable to describe any system and is especially useful for wave functions which undergo dramatic changes in the dominances of the Slater determinants as a consequence of the stretching of the internuclear distances within molecular systems.

This work has been organized as follows. In Sec. II, we review the main theoretical aspects involved in the proposed methodology, indicating the formulation of the $N$-electron seniority number operator by means of the spin-free secondorder replacement operators and the features of the CI expansions. Section III reports the computational details as well as the criteria followed to select the molecular orbital basis to perform this treatment. In Sec. IV, we show results obtained from the proposed hybrid method; in this work, the treatment involves all Slater determinants whose seniority number is zero, augmented with those of the single and double excitation configuration interaction (CISD) method not yet included. These results have been compared with those arising from the seniority-number approach (at zeroth seniority-number level), with those of the CISD or single, double, and triple excitation configuration interaction (CISDT) methods and with those of the FCI procedure. This section also reports the number of Slater determinants required in each method, in order to assess the computational expense. Finally in Sec. V, we summarize the main conclusions of this work.

\section{THEORETICAL REVIEW}

The $N$-electron seniority number operator $\hat{\Omega}$ can be formulated by means of the spin-free replacement operators $\mathrm{as}^{2}$

$$
\hat{\Omega}=\sum_{i=1}^{K}\left(\hat{E}_{i}^{i}-\hat{E}_{i i}^{i i}\right)
$$

in which $\hat{E}_{i}^{i}=\sum_{\sigma} a_{i}^{\dagger} a_{i} \sigma$ and $\hat{E}_{i i}^{i i}=\sum_{\sigma_{1}, \sigma_{2}} a_{i \sigma_{1}}^{\dagger} a_{i \sigma_{2}}^{\dagger} a_{i} \sigma_{2} a_{i} \sigma_{1}$ are the spin-free first- and second-order replacement operators, respectively. ${ }^{13-15}$ In this formalism, $a_{i \sigma}^{\dagger}$ and $a_{i} \sigma$ denote the standard creation and annihilation fermion operators corresponding to a spin-orbital $i^{\sigma}$, where $\sigma=\alpha, \beta$ means the spin coordinate and $i$ represents an orbital of an orthonormal basis set composed of $K$ functions. According to Eq. (1), the expectation value of the operator $\hat{\Omega}$ for an $N$-electron Slater determinant $\langle\hat{\Omega}\rangle=\Omega$ is the difference between the total number of electrons $N$ (i.e., $\sum_{i=1}^{K}\left\langle\hat{E}_{i}^{i}\right\rangle$ ) and the number of electrons corresponding to doubly occupied orbitals in the determinant $\left(\sum_{i=1}^{K}\left\langle\hat{E}_{i i}^{i i}\right\rangle\right)$. In other words, $\Omega$ is a positive integer meaning the number of unpaired electrons in the determinant.

The $N$-electron Slater determinants constructed with $K$ basis functions and a determined spin quantum number $S_{z}$ can be classified according to the values $\Omega=0,2,4 \ldots$ (for $N$ even) or $\Omega=1,3,5 \ldots$ (for $N$ odd), satisfying the constraint condition $\Omega \geq 2\left|S_{z}\right|$. In both cases, the maximum value of the $\Omega$ quantity is $\Omega_{\max }=N$ (if $K \geq N$ ) or $\Omega_{\max }=2 K-N$ (if $K<N$ ). The projection of the Hamiltonian operator corresponding to an $\mathrm{N}$-electron system on one or more sets of determinants (each set including all determinants with a given $\Omega$ value) yields an $N$-electron CI Hamiltonian matrix. The diagonalization of this matrix provides the eigenvalues and eigenvectors which are the energies and wave functions of the seniority-numberbased CI method at levels $\Omega=0,2,4 \ldots$ or $\Omega=1,3,5 \ldots$. In the limit when all possible values of $\Omega$ are taken into account $\left(\Omega=2\left|S_{z}\right|, 2\left(\left|S_{z}\right|+1\right), \ldots, \Omega_{\max }\right)$, the seniority-numberbased $\mathrm{CI}$ energies and wave functions coincide with those arisen from the FCI method, which utilize all possible determinants with a particular $S_{z}$ value, constructed with the $K$ functions, to perform the Hamiltonian projection. This $\operatorname{CI}(\Omega)$ procedure constitutes an alternative to the traditional one, in which the classification of the Slater determinants to project the Hamiltonian is implemented according to the excitation levels with respect to a reference determinant. ${ }^{16-19}$ Both CI approaches lead to different energies, although they converge to the FCI result when a high number of determinants is used in the numerical determinations. Furthermore, a basisset transformation yields changes in the results arising from both CI methods ${ }^{4}$ what makes it necessary to properly select the type of molecular orbitals leading to more efficient wave function expansions. ${ }^{1,4,20,21}$

The concept of seniority number has also been extended to $N$-electron spin-adapted wave functions $\Psi(N, S)$ with a given spin quantum number $S$ and any $S_{z}{ }^{2}$ In this case, the expectation value of the seniority number is

$$
\begin{aligned}
\langle\hat{\Omega}\rangle_{\Psi(N, S)} & =\langle\Psi(N, S)|\hat{\Omega}| \Psi(N, S)\rangle \\
& =\sum_{i}{ }^{1} D_{i}^{i}-2 \sum_{i}{ }^{2} D_{i i}^{i i}
\end{aligned}
$$

in which ${ }^{1} D_{i}^{i}=\left\langle\Psi(N, S)\left|\hat{E}_{i}^{i}\right| \Psi(N, S)\right\rangle$ and ${ }^{2} D_{i i}^{i i}=\frac{1}{2}\langle\Psi(N, S)|$ $\hat{E}_{i i}^{i i}|\Psi(N, S)\rangle$ are elements of the spin-free first- and secondorder reduced density matrices, respectively, corresponding to the wave function $\Psi(N, S)$. In this formulation, the $S_{z}$ quantum number has been omitted, since these matrix elements are independent of the spin projection. The expectation value $\langle\hat{\Omega}\rangle_{\Psi(N, S)}$ is no longer an integer like in a determinant case but a quantity depending on the nature of the $i$ orbitals. In Refs. 2 and 4, we have proposed to perform transformations of the molecular basis set so that the expectation value of the seniority number operator for a FCI state, $\langle\hat{\Omega}\rangle_{\Psi(N, S)}$, gets a minimum value. The computational procedure we have used, based on the method reported by Subotnik et al., ${ }^{22}$ allows one to obtain more compact FCI wave functions and CI wave functions attaining larger correlation energies. The orbitals resulting from that minimization have been denominated $\mathrm{M}_{\min }$ in contrast to the natural orbitals (NO) and the HartreeFock canonical molecular orbitals (CMO). The functions that constitute the basis sets $M_{\min }$ are not, in general, symmetryadapted orbitals although the molecular symmetry can be restored. Other applications of the expectation value $\langle\hat{\Omega}\rangle_{\Psi(N, S)}$ arise from its relationship with the effectively unpaired electron number corresponding to the state $\Psi(N, S) .23,24$

As has been mentioned in the Introduction, the excitationbased and the seniority-number-based CI expansions present advantages and deficiencies, respectively, to describe systems requiring a simple determinant as zeroth-order wave functions, but both of them show the opposite behavior when 
multideterminantal wave functions are needed at this level of approximation. ${ }^{1}$ There are ambiguous situations in which a system is suitably described by expansions with a very strong dominance of a Slater determinant at a determined geometrical arrangement but requires several Slater determinants at other arrangements. Consequently, it seems reasonable to study the performance of the Hamiltonian projection on the union of the sets of determinants from the seniority-number-based and excitation-based CI methods (at given $\Omega$ and excitation levels). This configuration space is wider than that constituted by each CI method individually and necessarily provides better results at any geometrical arrangement. CI expansions of hybrid character based on excitations and other features of the determinants (energies) have also been recently proposed. ${ }^{25}$ In Secs. III-V, we describe results drawn out at several geometrical arrangements of systems which present marked changes in the dominances of the Slater determinants.

\section{COMPUTATIONAL DETAILS}

The reliability of our methodology has been tested by determining ground-state electronic energies in closed-shell atomic and molecular systems using the hybrid CI scheme. We have projected the Hamiltonian of these systems on the union of the Slater determinant sets required in the $\mathrm{CI}(\Omega=0)$ and CISD procedures, what has been denoted as $\mathrm{CI}_{\mathrm{SD}}^{(\Omega=0)}$. The dimensions of this $N$-electron Hamiltonian matrix is $\sum_{i=0}^{2} \sum_{p=0}^{i}\left(\begin{array}{c}K-\frac{N}{2} \\ p\end{array}\right)\left(\begin{array}{c}\frac{N}{2} \\ p\end{array}\right)\left(\begin{array}{c}K-\frac{N}{2} \\ i-p\end{array}\right)\left(\begin{array}{c}\frac{N}{2} \\ i-p\end{array}\right)+\left(\begin{array}{c}K \\ \frac{N}{2}\end{array}\right)$ $-\left[\frac{N}{2}\left(K-\frac{N}{2}\right)+1\right]$, in which the first term is the CISD Hamiltonian matrix size, the second term is the dimension of the $\Omega=0$ Hamiltonian matrix, and the last one in the number Slater determinants belonging to both spaces. The results have been compared with those arising from the individual $\mathrm{CI}(\Omega=0)$, CISD, and CISDT procedures, as well as with those provided by the FCI method. The excitation level of the determinants used in the CISD, CISDT, etc., procedures has been evaluated with respect to a single reference determinant. In the molecular calculations, we have used small basis sets in order to get FCI results at an affordable cost. The basis sets utilized for each system have been indicated in the tables and figures in Sec. IV. We have carried out the corresponding basis set transformations so that finally the $N$-electron CI matrices have been expressed in the $\mathrm{M}_{\min }$ orbitals in which the expectation values $\langle\hat{\Omega}\rangle_{\Psi(N, S)}$ attain the minimum values for

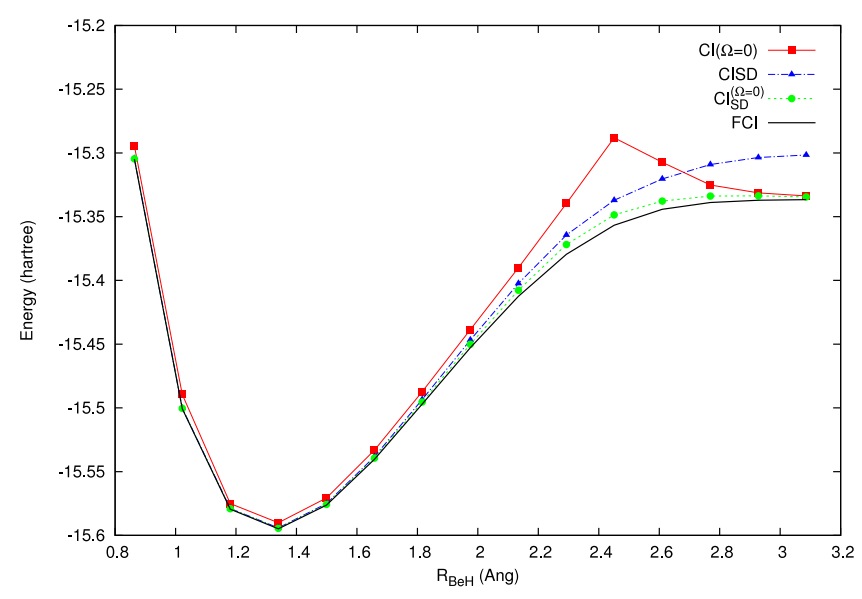

FIG. 1. Potential energy curves for the symmetric dissociation of the $\mathrm{BeH}_{2}$ molecule using STO-3G basis set transformed to the basis which minimizes the seniority number for the FCI procedure $\left(\mathrm{M}_{\min }\right)$.

the FCI procedure. ${ }^{4}$ Experimental equilibrium bond lengths and angles and symmetrically stretched configurations have been used to describe potential energy curves in the molecular systems. The one- and two-electron integrals required to construct the $N$-electron CI matrices have been obtained from a modified version of the PSI 3.3 package. ${ }^{26}$ We have also used that code to calculate the Hartree-Fock CMOs utilized as initial basis in the iterative procedure which determines the $M_{\text {min }}$ basis sets. In subsequent steps, we have elaborated our own codes to perform the basis set transformations and to construct the $N$-electron $\mathrm{CI}(\Omega=0), \mathrm{CISD}, \mathrm{CI}_{\mathrm{SD}}^{(\Omega=0)}$, and CISDT matrices; this last task has been implemented using the algorithms in the programmes developed for Refs. 27 and 28.

\section{RESULTS AND DISCUSSION}

Table I gathers energy values of selected atomic systems arising from the $\mathrm{CI}(\Omega=0), \mathrm{CISD}, \mathrm{CI}_{\mathrm{SD}}^{(\Omega=0)}$, CISDT, and FCI methods, as well as their computational cost. This cost has been reflected by means of the number of Slater determinants in the Hamiltonian projections. This table also shows the Gaussian basis sets used in these numerical determinations although all values reported in this table have been obtained from the corresponding $\mathbf{M}_{\min }$ orthonormal orbital basis. The $\mathrm{Be}$ atom has been chosen as prototype of system having a marked static correlation; ${ }^{1}$ in fact, the $\mathrm{CI}(\Omega=0)$ result is

TABLE I. Values of energies, computational cost, and basis sets utilized for atomic systems calculated at different levels of CI methods. Results were obtained using the orthonormal basis that minimizes the seniority number for the FCI procedure $\left(\mathrm{M}_{\min }\right)$.

\begin{tabular}{|c|c|c|c|c|c|c|c|c|c|c|c|}
\hline \multirow[b]{2}{*}{ System } & \multirow[b]{2}{*}{ Basis } & \multicolumn{5}{|c|}{ No. determinants } & \multicolumn{5}{|c|}{ Energy/ $E_{h}$} \\
\hline & & $\mathrm{CI}(\Omega=0)$ & CISD & $\mathrm{CI}_{\mathrm{SD}}^{(\Omega=0)}$ & CISDT & FCI & $\mathrm{CI}(\Omega=0)$ & CISD & $\mathrm{CI}_{\mathrm{SD}}^{(\Omega=0)}$ & CISDT & FCI \\
\hline $\mathrm{Be}$ & cc-pVDZ & 91 & 757 & 823 & 3925 & 8281 & -14.617064 & -14.617355 & -14.617365 & -14.617393 & -14.617409 \\
\hline $\mathrm{F}^{-}$ & cc-pVDZ & 2002 & 2836 & 4792 & 36916 & 4008004 & -99.430350 & -99.553918 & -99.555046 & -99.554522 & -99.560772 \\
\hline $\mathrm{Ne}$ & cc-pVDZ & 2002 & 2836 & 4792 & 36916 & 4008004 & -128.551000 & -128.675320 & -128.676110 & -128.676430 & -128.680896 \\
\hline $\mathrm{Mg}$ & $6-31 G$ & 1716 & 2479 & 4152 & 30339 & 2944656 & -199.627920 & -199.630130 & -199.630160 & -199.630240 & -199.630374 \\
\hline $\mathrm{Al}^{+}$ & $6-31 G$ & 1716 & 2479 & 4152 & 30339 & 2944656 & -241.690140 & -241.692590 & -241.692620 & -241.692690 & -241.692805 \\
\hline $\mathrm{Ar}$ & $6-31 G$ & 715 & 1801 & 2479 & 18025 & 511225 & -526.784570 & -526.812090 & -526.812220 & -526.812690 & -526.813352 \\
\hline
\end{tabular}




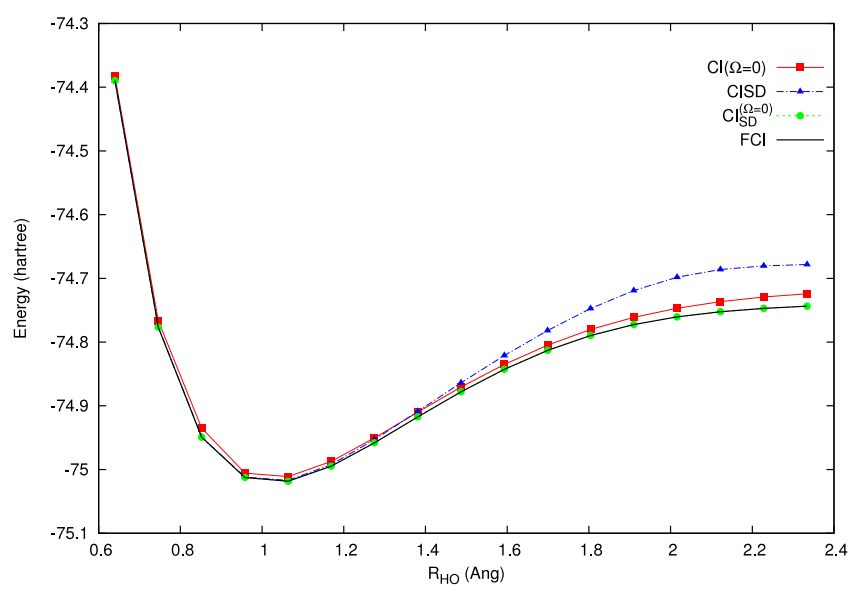

FIG. 2. Potential energy curves for the symmetric dissociation of the $\mathrm{H}_{2} \mathrm{O}$ molecule using STO-3G basis set transformed to the basis which minimizes the seniority number for the FCI procedure $\left(\mathrm{M}_{\min }\right)$.

close to the CISD one with lower computational cost (only 91 Slater determinants). The hybrid $\mathrm{CI}_{\mathrm{SD}}^{(\Omega=0)}$ method, proposed in this work, leads to a better result being very close to that provided by the CISDT procedure with a markedly lower computational cost (823 Slater determinants vs 3925 ones). The correlation energy in the $\mathrm{Ne}$ atom has been considered to be purely dynamic; ${ }^{1}$ our results confirm this behavior since the $\mathrm{CI}(\Omega=0)$ result is no longer close to the CISD value as in the Be case. However, the hybrid $\mathrm{CI}_{\mathrm{SD}}^{(\Omega=0)}$ method yields a value quite close to that of the CISDT method at lower computational cost and in the $\mathrm{F}^{-}$case (a system isoelectronic with the $\mathrm{Ne}$ atom) the $\mathrm{CI}_{\mathrm{SD}}^{(\Omega=0)}$ value turns out to be even lower than that obtained from the CISDT procedure. The systems $\mathrm{Mg}$ and $\mathrm{Al}^{+}$ can be classified between those possessing an intermediate strength of static correlation energy; the hybrid $\mathrm{CI}_{\mathrm{SD}}^{(\Omega=0)}$ method also predicts satisfactory results for these systems. The results found for the Ar atom show that this system presents a behavior similar to that of the Ne atom; both atoms possess essentially dynamic correlation, as expected.

In Figs. 1-3, we show potential energy curves of the symmetrically stretched systems $\mathrm{BeH}_{2}$ and $\mathrm{H}_{2} \mathrm{O}$ as well as that

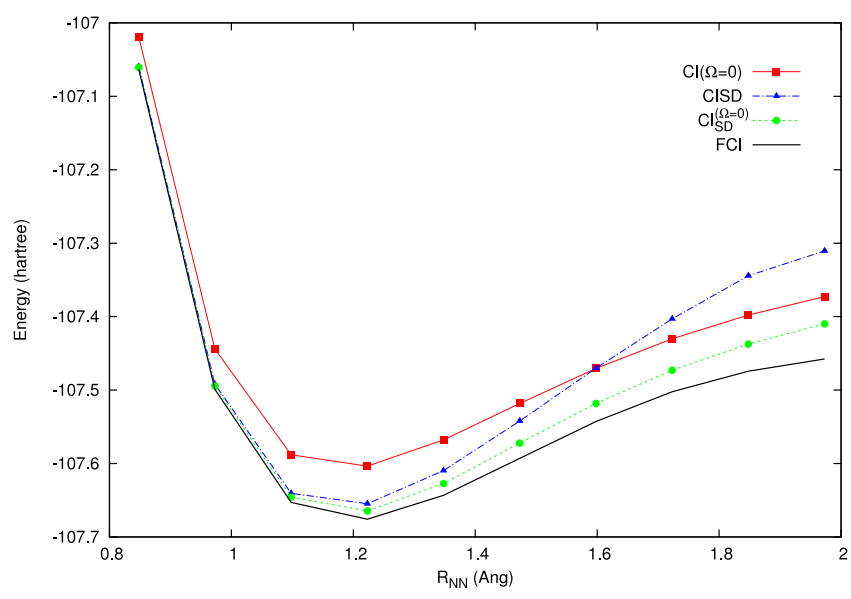

FIG. 3. Potential energy curves for the dissociation of the $\mathrm{N}_{2}$ molecule using STO-3G basis set transformed to the basis which minimizes the seniority number for the FCI procedure $\left(\mathrm{M}_{\min }\right)$.
TABLE II. MAE and NPE (in $m E_{h}$ ) of the CI potential energy curves with respect the FCI one. Results were obtained using the orthonormal basis set that minimizes the seniority number for the FCI procedure $\left(\mathrm{M}_{\min }\right)$.

\begin{tabular}{|c|c|c|c|c|c|c|}
\hline & \multicolumn{2}{|c|}{$\mathrm{CI}(\Omega=0)$} & \multicolumn{2}{|c|}{ CISD } & \multicolumn{2}{|c|}{$\mathrm{CI}_{\mathrm{SD}}^{(\Omega=0)}$} \\
\hline & MAE & NPE & MAE & NPE & MAE & NPE \\
\hline $\mathrm{BeH}_{2}$ & 68.60 & 65.73 & 35.05 & 34.61 & 8.12 & 7.84 \\
\hline $\mathrm{H}_{2} \mathrm{O}$ & 19.53 & 12.85 & 66.69 & 66.60 & 0.19 & 0.17 \\
\hline $\mathrm{N}_{2}$ & 84.79 & 40.75 & 147.1 & 143.3 & 47.71 & 45.18 \\
\hline
\end{tabular}

of the $\mathrm{N}_{2}$ molecule. Our aim is to assess the performance of the $\mathrm{CI}_{\mathrm{SD}}^{(\Omega=0)}$ method by comparing its results and computational cost with those arising from the more known $\mathrm{CI}(\Omega=0)$, CISD, and FCI procedures. Each numerical value described in these curves has been obtained using the basis set $\mathrm{M}_{\min }$ in which the $\langle\hat{\Omega}\rangle_{\Psi(N, S)}$ quantity gets a minimum value for the corresponding FCI wave function $\Psi(N, S)$. The accuracy of the potential energy curves obtained from the CI methods with respect to the FCI one is compared in Table II, where the maximum absolute errors (MAE) and nonparallelity errors (NPE) are shown for each method. As can be observed, the $\mathrm{CI}_{\mathrm{SD}}^{(\Omega=0)}$ method decreases these quantities in one and two orders of magnitude for the $\mathrm{BeH}_{2}$ and $\mathrm{H}_{2} \mathrm{O}$ systems and near one order of magnitude for the $\mathrm{N}_{2}$ molecule. The computational cost of these numerical determinations has been reflected in Table III in terms of the number of Slater determinants required in each method. A survey of the $\mathrm{BeH}_{2}$ curve shapes described in Fig. 1 shows very good agreement for all studied methods at near-equilibrium distances. However, at longer internuclear distances, where static correlation is higher, the $\mathrm{CI}(\Omega=0)$ method exhibits a hump, which has also been reported and discussed in other systems $;{ }^{1}$ although near the dissociation, this curve converges to the FCI one. This hump is no longer present in the results yielded by the hybrid $\mathrm{CI}_{\mathrm{SD}}^{(\Omega=0)}$ method which requires only a little more computational effort compared to the CISD procedure. As expected, the $\mathrm{CI}_{\mathrm{SD}}^{(\Omega=0)}$ method agrees better with the FCI one in the whole interval of internuclear distances studied. Fig. 2 describes the potential energy curves of the $\mathrm{H}_{2} \mathrm{O}$ molecule. As can be observed, the results from the $\mathrm{CI}_{\mathrm{SD}}^{(\Omega=0)}$ method are near coincident with the FCI ones both for near-equilibrium geometries as well as for distances close to the dissociation limit, although the computational cost is only slightly higher than the CISD one. In Fig. 3, we show the results corresponding to the $\mathrm{N}_{2}$ molecule in which we appreciate the crossing between the $\mathrm{CI}(\Omega=0)$ and CISD curves. The CISD values are closer to the FCI ones than those of $\mathrm{CI}(\Omega=0)$ method at the near-equilibrium region (low static correlation)

TABLE III. Computational cost reflected in the size of the Slater determinant basis for molecular systems calculated at different levels of CI methods.

\begin{tabular}{lccccr}
\hline \hline & & \multicolumn{4}{c}{ No. determinants } \\
\cline { 3 - 6 } System & Basis & $\mathrm{CI}(\Omega=0)$ & $\mathrm{CISD}$ & $\mathrm{CI}_{\mathrm{SD}}^{(\Omega=0)}$ & FCI \\
\hline $\mathrm{BeH}_{2}$ & STO-3G & 35 & 205 & 227 & 1225 \\
$\mathrm{H}_{2} \mathrm{O}$ & STO-3G & 21 & 141 & 151 & 441 \\
$\mathrm{~N}_{2}$ & STO-3G & 120 & 610 & 708 & 14400 \\
\hline \hline
\end{tabular}




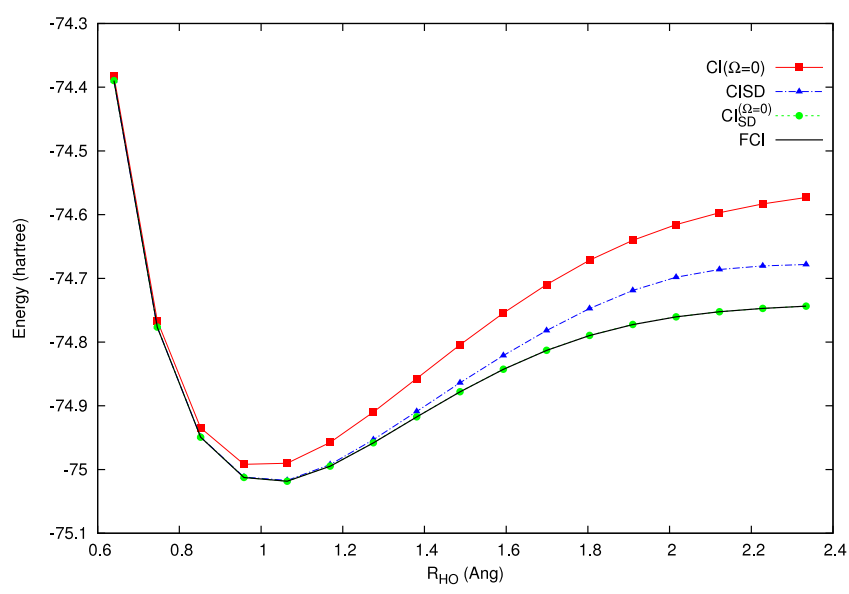

FIG. 4. Potential energy curves for the symmetric dissociation of the $\mathrm{H}_{2} \mathrm{O}$ molecule using STO-3G basis set transformed to the NO set which diagonalizes the FCI first-order reduced density matrix.

but this behavior is quite opposite at the dissociation limit region (high static correlation). Similarly to the $\mathrm{BeH}_{2}$ and $\mathrm{H}_{2} \mathrm{O}$ molecules, the performance of the $\mathrm{CI}_{\mathrm{SD}}^{(\Omega=0)}$ curve in the $\mathrm{N}_{2}$ system is better than those provided by the $\operatorname{CI}(\Omega=0)$ and CISD methods individually and its computational cost is only slightly higher than that required by the CISD procedure.

We have studied the behavior of the results provided by the $\mathrm{CI}_{\mathrm{SD}}^{(\Omega=0)}$ method using molecular orbital basis sets other than the $\mathrm{M}_{\min }$ ones. In Figs. 4 and 5, we have represented potential energy curves of the symmetrically stretched $\mathrm{H}_{2} \mathrm{O}$ molecule using the $\mathrm{NO}$ and $\mathrm{CMO}$ basis sets, respectively. As can be seen by comparing Figs. 2 and 4 , the $\operatorname{CI}(\Omega=0)$ results are closer to the FCI ones in the $\mathrm{M}_{\min }$ basis set, in agreement with the conclusions reported in Ref. 4, providing better results than the CISD ones. However, the $\mathrm{CI}_{\mathrm{SD}}^{(\Omega=0)}$ curve with the NO basis set (Fig. 4) is just as nearly coincident with the FCI one as the $\mathrm{CI}_{\mathrm{SD}}^{(\Omega)=0)}$ curve with the $\mathrm{M}_{\min }$ basis (Fig. 2). The results arising from the use of the CMOs, shown in Fig. 5, also indicate the closeness of the $\mathrm{CI}_{\mathrm{SD}}^{(\Omega=0)}$ curve to the FCI one. From a computational point of view, the $\mathrm{CMO}$ basis sets are more easily available than the $\mathrm{NO}$ and $\mathrm{M}_{\min }$ ones, and consequently, they could be preferably used within the $\mathrm{CI}_{\mathrm{SD}}^{(\Omega=0)}$ scheme, avoiding

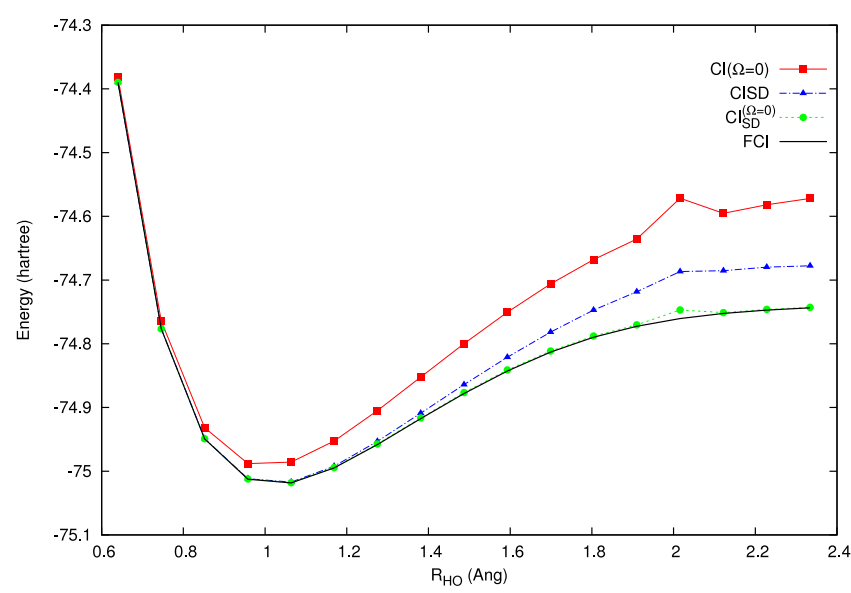

FIG. 5. Potential energy curves for the symmetric dissociation of the $\mathrm{H}_{2} \mathrm{O}$ molecule using STO-3G basis set transformed to the Hartree-Fock CMO set.

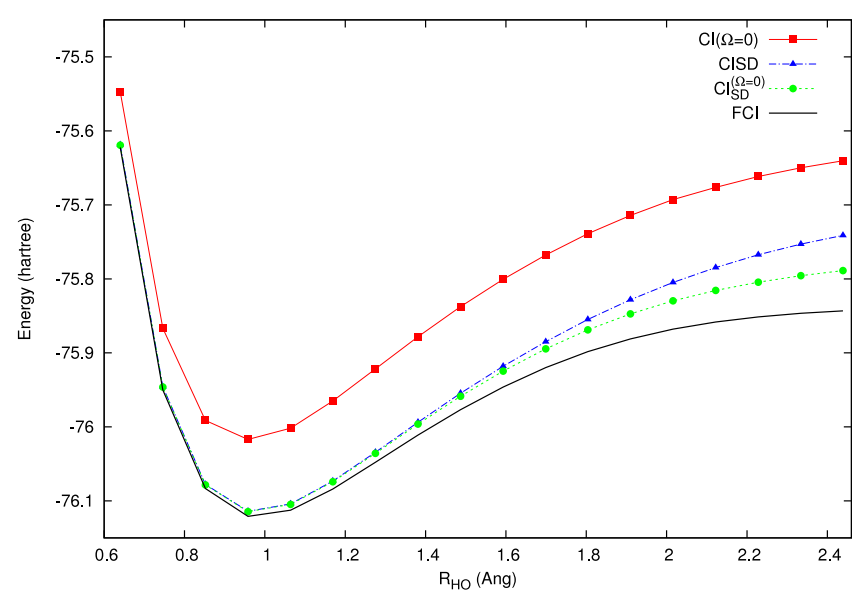

FIG. 6. Potential energy curves for the symmetric dissociation of the $\mathrm{H}_{2} \mathrm{O}$ molecule using 6-31G basis set transformed to the Hartree-Fock CMO set.

the FCI determinations. Fig. 5 also shows the presence of a small hump in the curves $\mathrm{CI}(\Omega=0), \mathrm{CISD}$, and $\mathrm{CI}_{\mathrm{SD}}^{(\Omega=0)}$ in the CMO basis set, which does not appear in their counterparts calculated in the $\mathrm{NO}$ and $\mathrm{M}_{\text {min }}$ basis sets. However, the hump in the $\mathrm{CMO}$ curves gets its smallest size in the $\mathrm{CI}_{\mathrm{SD}}^{(\Omega=0)}$ method. In order to elucidate whether the hump is an artefact of using a minimal basis set, we also performed calculations using the split valence 6-31G basis set, revealing indeed that the use of a larger basis set leads to the disappearance of this hump (see Fig. 6). Also note that all main features reported for the STO$3 \mathrm{G}$ data remain for the larger basis set.

Truncated methods, i.e., methods other than FCI one, suffer from an undesirable dependence on the basis chosen, as is clearly demonstrated above. We, therefore, also performed preliminary calculations at $\mathrm{CI}_{\mathrm{SD}}^{(\Omega=0)}$ level, with an energy optimized single particle basis to examine the magnitude of the further change in energy compared to the energies obtained using the other molecular orbital bases. We have chosen the $\mathrm{N}_{2}$ molecule as a prototype system. Although a detailed description of the algorithm used and more detailed examinations of this effect will be presented in future work, Fig. 7 clearly shows that the energy optimized basis set

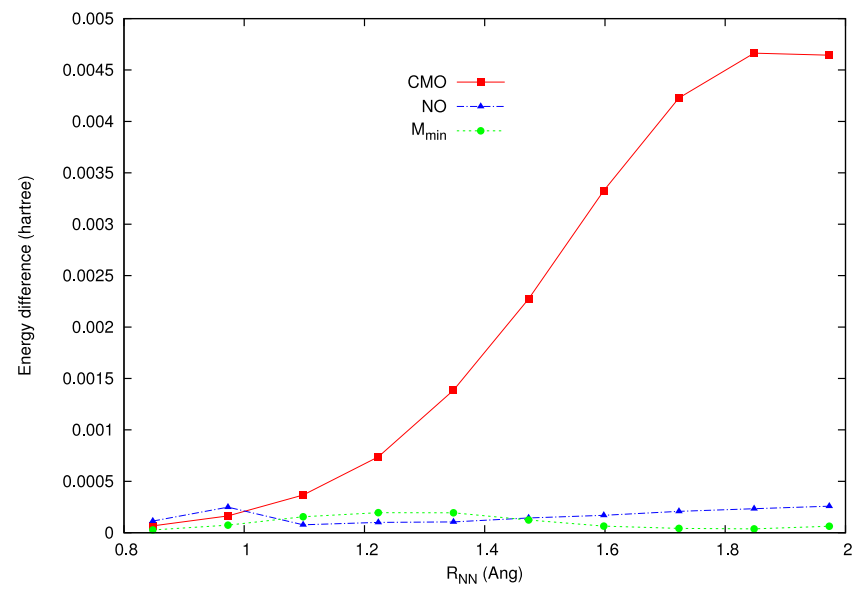

FIG. 7. $\mathrm{CI}_{\mathrm{SD}}^{(\Omega=0)}$ energy differences with respect to the energy optimized basis set for different single particle bases as a function of internuclear distance in the $\mathrm{N}_{2}$ molecule. 


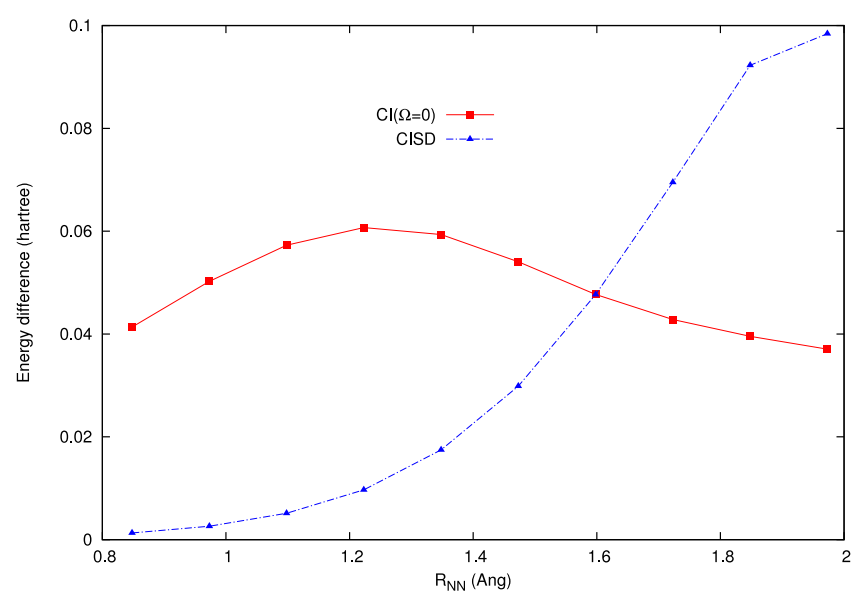

FIG. 8. Energy differences for $\mathrm{CI}(\Omega=0)$ and CISD methods with respect to the $\mathrm{CI}_{\mathrm{SD}}^{(\Omega=0)}$ model using energy optimized orbitals for each method as a function of internuclear distance in the $\mathrm{N}_{2}$ molecule.

slightly improves on the energies calculated using the NO and $\mathrm{M}_{\text {min }}$ basis sets and that these energies are better than those given by the CMO basis set although their respective order may differ along the bond stretching coordinate. The key conclusion of the paper is, however, confirmed in all cases: as shown in Fig. 8, the union of the $\operatorname{CI}(\Omega=0)$ and CISD determinant sets lowers significantly the energies provided by those procedures individually without an important increase in computational cost.

\section{CONCLUDING REMARKS AND PERSPECTIVES}

In this work, we have proposed a new procedure which possesses a hybrid character within the CI technique framework. The method is based on the projection of an $N$-electron Hamiltonian on the union of two Slater determinant sets, each of them constituted according to a different criterion. One of these sets is composed of all Slater determinants up to a determined seniority number; the other set is composed of a reference determinant and its excitations up to a determined level. We report numerical determinations on selected atomic and molecular systems using a simple version of the hybrid method, the $\mathrm{CI}_{\mathrm{SD}}^{(\Omega=0)}$ one. Its results and computational cost are compared with those obtained from its parents $\operatorname{CI}(\Omega=0)$ and CISD methods. Our proposal turns out to be particularly useful to describe systems which undergo strong changes in the static correlation energies at stretched geometries with respect to near-equilibrium arrangements, correcting the deficiencies of the $\mathrm{CI}(\Omega=0)$ and CISD procedures. Notably, the sets of the $\operatorname{CI}(\Omega=0)$ and the CISD Slater determinants act like ideal complements to each other, solving many of the issues related to strong static correlation. The NO and $\mathrm{M}_{\text {min }}$ basis sets are optimal for the traditional and senioritytype CI procedures, respectively, from a compactness of wave function perspective, and consequently, we have considered these basis sets in our calculations. Our best results have been obtained using the $\mathrm{M}_{\text {min }}$ molecular orbital basis sets, which minimize the seniority number for the FCI procedure. However, we also show that other molecular basis sets, as those of CMOs, improve the $\mathrm{CI}(\Omega=0)$ and CISD results with lower computational costs. One can also find the optimal molecular orbital basis sets for any CI-type method from an energy perspective. We also report preliminary calculations with a $\mathrm{CI}_{\mathrm{SD}}^{(\Omega=0)}$ energy optimized molecular orbital basis set pointing out a further energy lowering. Work along this line is being pursued in our laboratories.

\section{ACKNOWLEDGMENTS}

This work has been financially supported by the Project Nos. PIP 11220090100061, 11220090100369, and 11220090100546 (Consejo Nacional de Investigaciones Científicas y Técnicas); UBACYT 20020100100197 (Universidad de Buenos Aires); PICT 2011-01217 (Agencia Nacional de Promoción Científica y Tecnológica); GIU12/09 and UFI11/07 (Universidad del Pais Vasco); the FWO-MINCyT international collaboration Grant No. VS.0001.14N/FW/13/03 and the special research fund of Ghent University. We thank the Universidad del Pais Vasco and the Universidad de Buenos Aires for allocation of computational resources.

${ }^{1}$ L. Bytautas, T. M. Henderson, C. A. Jiménez-Hoyos, J. K. Ellis, and G. E. Scuseria, J. Chem. Phys. 135, 044119 (2011).

${ }^{2}$ D. R. Alcoba, A. Torre, L. Lain, G. E. Massaccesi, and O. B. Oña, J. Chem. Phys. 139, 084103 (2013).

${ }^{3}$ K. Boguslawski, P. Tecmer, P. A. Limacher, P. A. Johnson, P. W. Ayers, P. Bultinck, S. Baerdemacker, and D. Van Neck, J. Chem. Phys. 140, 214114 (2014).

${ }^{4}$ D. R. Alcoba, A. Torre, L. Lain, G. E. Massaccesi, and O. B. Oña, J. Chem. Phys. 140, 234103 (2014).

${ }^{5}$ P. A. Limacher, T. D. Kim, P. W. Ayers, P. A. Johnson, S. De Baerdemacker, D. Van Neck, and P. Bultinck, Mol. Phys. 112, 853 (2014).

${ }^{6} \mathrm{P}$. Ring and P. Schuck, The Nuclear Many-Body Problem (Springer-Verlag, New York, 1980).

${ }^{7}$ D. S. Koltun and J. M. Eisenberg, Quantum Mechanics of Many Degrees of Freedom (Wiley, New York, 1988).

${ }^{8}$ A. J. Garza, C. A. Jiménez-Hoyos, and G. E. Scuseria, J. Chem. Phys. 138, 134102 (2013).

${ }^{9}$ P. A Limacher, P. W. Ayers, P. A. Johnson, S. De Baerdemacker, D. Van Neck, and P. Bultinck, J. Chem. Theory and Comput. 9, 1394 (2013).

${ }^{10}$ C. A. Jiménez-Hoyos, R. Rodríguez-Guzmán, and G. E. Scuseria, J. Chem. Phys. 139, 204102 (2013).

${ }^{11}$ L. M. Mentel, R. van Meer, O. V. Gritsenko, and E. J. Baerends, J. Chem. Phys. 140, 214105 (2014).

${ }^{12}$ T. Stein, T. M. Henderson, and G. E. Scuseria, J. Chem. Phys. 140, 214113 (2014).

${ }^{13}$ J. Paldus and B. Jeziorski, Theor. Chim. Acta 73, 81 (1988).

${ }^{14}$ A. Torre, L. Lain, and J. Millan, Phys. Rev. A 47, 923 (1993).

${ }^{15}$ L. Lain and A. Torre, Phys. Rev. A 52, 2446 (1995).

${ }^{16}$ E. R. Davidson, Rev. Mod. Phys. 44, 451 (1972).

${ }^{17}$ I. Shavitt, B. J. Rosenberg, and S. Palakit, Int. J. Quantum Chem. 10(S10), 33 (1976).

${ }^{18}$ I. Shavitt, Mol. Phys. 94, 3 (1998)

${ }^{19}$ C. D. Sherrill and H. F. Schaefer III, Adv. Quantum Chem. 34, 143 (1999).

${ }^{20}$ D. H. Kobe, J. Chem. Phys. 50, 5183 (1969).

${ }^{21}$ K. J. H. Giesbertz, Chem. Phys. Lett. 591, 220 (2014).

${ }^{22}$ J. E. Subotnik, Y. Shao, W. Liang, and M. Head-Gordon, J. Chem. Phys. 121, 9220 (2004).

${ }^{23}$ D. R. Alcoba, R. C. Bochicchio, L. Lain, and A. Torre, Chem. Phys. Lett. 429, 286 (2006).

${ }^{24}$ P. Karafiloglou, J. Chem. Phys. 130, 164103 (2009).

${ }^{25}$ F. A. Evangelista, J. Chem. Phys. 140, 124114 (2014).

${ }^{26}$ T. D. Crawford, C. D. Sherrill, E. F. Valeev, J. T. Fermann, R. A. King, M. L. Leininger, S. T. Brown, C. L. Janssen, E. T. Seidl, J. P. Kenny, and W. D. Allen, J. Comput. Chem. 28, 1610 (2007).

${ }^{27}$ G. Knizia and G. K.-L. Chan, Phys. Rev. Lett. 109, 186404 (2012).

${ }^{28}$ G. Knizia and G. K.-L. Chan, J. Chem. Theory Comput. 9, 1428 (2013). 